\title{
Transcriptomic Evidence of Adaptive Evolution of the Epiphytic Fern Asplenium nidus
}

\author{
Jiao Zhang $\mathbb{D}^{1,2,3}$ Li Liu $\mathbb{D}$, ${ }^{1}$ Jiang-Ping Shu $\mathbb{D}^{1},{ }^{1}$ Dong-Mei Jin $\mathbb{D}^{1},{ }^{1}$ Hui Shen $\left(\mathbb{D},{ }^{1}\right.$ \\ Hong-Feng Chen $\mathbb{D}^{4}{ }^{4}$ Rui Zhang $\mathbb{D}^{1},{ }^{1}$ and Yue-Hong Yan $\mathbb{D}^{1,2}$ \\ ${ }^{1}$ Shanghai Chenshan Plant Science Research Center, Shanghai Chenshan Botanical Garden, Chinese Academy of Sciences, \\ Shanghai 201602, China \\ ${ }^{2}$ Shanghai Chenshan Plant Science Research Center, Institute of Plant Physiology and Ecology, Shanghai Institutes for \\ Biological Sciences, Chinese Academy of Sciences, Shanghai 200032, China \\ ${ }^{3}$ University of Chinese Academy of Sciences, Beijing 100049, China \\ ${ }^{4}$ Key Laboratory of Plant Resources Conservation and Sustainable Utilization, South China Botanical Garden, Chinese Academy \\ of Sciences, Guangzhou 510650, China
}

Correspondence should be addressed to Rui Zhang; zhangrui@csnbgsh.cn and Yue-Hong Yan; yhyan@sibs.ac.cn

Received 24 June 2019; Accepted 11 October 2019; Published 1 December 2019

Academic Editor: Marco Gerdol

Copyright (c) 2019 Jiao Zhang et al. This is an open access article distributed under the Creative Commons Attribution License, which permits unrestricted use, distribution, and reproduction in any medium, provided the original work is properly cited.

\begin{abstract}
Epiphytic ferns have been found to flourish after angiosperms dominated forest communities, and they play important roles in rainforest canopies. How do epiphytic ferns adapt to tropical rainforest canopy habitats? At present, we know little about the molecular mechanism underlying this adaptation. Asplenium nidus is a well-known epiphytic fern that is closely related to the terrestrial species Asplenium komarovii. Here, RNA-seq and comparative transcriptomic analyses were performed to explore the underlying basis of the adaptation of $A$. nidus to extreme environments. A total of 44.04 and $44.57 \mathrm{Mb}$ clean reads were obtained from A. nidus and A. komarovii, respectively, and they were assembled into 89,741 and 77,912 unigenes. Functional annotation showed that 52,305 (58.28\% of the total genes for A. nidus) and 45,938 (58.96\% of the total genes for A. komarovii) unigenes were annotated in public databases. Genes involved in stress responses and photosynthesis were found to have undergone positive selection in A. nidus. Compared to A. komarovii, transcription factors related to stress response, leaf development, and root development were found to be considerably expanded in A. nidus, especially in the ANR1 subclade of MADS-box family genes which played roles in lateral root development. This study improves our understanding of the adaptation of $A$. nidus to epiphytic habitats by forming unique strategies.
\end{abstract}

\section{Introduction}

With 20,000-25,000 species, vascular epiphytes present widely in many families in ferns, gymnosperms, and angiosperms [1, 2]. Epiphytes play important ecological roles in rainforests [3, 4]. Ferns are the second largest group of vascular plants, of which 2,800 species are epiphytic. As a unique group, epiphytic ferns account for one-third of the leptosporangiate ferns, and they can endure severe drought stress, nutrient shortage, and intense sunlight, but not frost $[1,5]$. Several epiphytic ferns, including some species in the genera Vittaria, Pyrrosia, Polypodium, and Platycerium, have been found to exhibit features typical of crassulacean acid metabolism (CAM) in photosynthesis [6-8]. Epiphytic plants utilize specialized ecological strategies to adapt to variable environments, especially drought stress during dry seasons.

A. nidus is a well-known C3 epiphytic fern, and phylogenetic analyses have revealed that it belongs to the Neottopteris clade, which is the sister of the Phyllitis clade containing the terrestrial species A. komarovii (A. scolopendrium L. subsp. Japonicum) [9-11]. These two species have considerable similarities in morphology, including the presence of lanceolate simple leaves spirally tightly clustered into a bird's nest fern. However, A. nidus usually grows to a large size on trees or rocks within the canopy, while A. komarovii is not 
epiphytic and remains small, growing only on the forest floor. The close relatedness but difference in habitat makes these ferns ideal models for comparative genomics and evolution studies of epiphytes.

Comparative genomics (transcriptomics) involves characterizing the differences in gene expression, and it is commonly used in studies of adaptive evolution or evolutionary developmental biology [12-14]. Moreover, transcriptomic data are widely used to study nonmodel organisms [15-17]. In this study, we generated and annotated de novo transcriptome assemblies for both $A$. nidus and A. komarovii. Comparative analyses were performed to identify (1) unique genes expressed by $A$. nidus, (2) gene families that have undergone significant expansion, and (3) genes that are under positive selection. We aim to provide new insights on the transcriptomic mechanisms by which epiphytic ferns adapt to canopy habitats in tropical rainforests.

\section{Materials and Methods}

2.1. Sample Collection and Transcriptome Sequencing. A. nidus and $A$. komarovii were cultivated in a greenhouse of Shanghai Chenshan Botanical Garden (Shanghai, China). Young sporophylls of each species were collected and snap frozen in liquid nitrogen. High-quality total RNA was extracted using TRIzol reagent (Thermo Fisher Scientific, Waltham, MA, USA) following the manufacturer's instructions [18]. After cDNA library preparation, RNA-seq was performed on the Illumina HiSeq 2500 platform at the Beijing Genome Institute (Shenzhen, China). Raw reads of both $A$. nidus and A. komarovii were deposited into the NCBI short read archive under accession numbers SAMN11175064 and SAMN11175065.

2.2. De Novo Assembly and Functional Annotation. Raw reads were filtered using the Dynamic Trim function in SolexaQA [19] at a quality threshold of 20. Reads shorter than $50 \mathrm{bp}$ were removed. After the filtering, 6.61 and $6.69 \mathrm{G}$ of clean data were obtained from $A$. nidus and $A$. komarovii, respectively. Trinity [20] was used for de novo transcriptome assembly with default settings except that "min_kmer-cov $=2$ " to reduce the number of errorcontaining kmers. Thereafter, TGICL 2.1 [21] was utilized to remove the redundant contigs.

The largest contigs were treated as candidate unigenes and used for subsequent analyses. All unigenes were annotated by searching NCBI nonredundant protein database $(\mathrm{Nt})$, the NCBI nonredundant nucleotide database $(\mathrm{Nr})$, the NCBI nonredundant nucleotide database $(\mathrm{Nr})$, the Swiss Institute of Bioinformatics (Swiss-Prot), InterPro, the Gene Ontology database (GO), and the Kyoto Encyclopedia of Genes and Genomes (KEGG). BLAST searches were performed against the $\mathrm{Nt}, \mathrm{Nr}$ and the Swiss-Prot databases with an $e$ value cut-off of $10-5$. Thereafter, protein domains were annotated using InterProScan5 [22]. GO annotation was performed by Blast2GO [23]. Transcription factors (TFs) were identified by HMM v3 [24] querying of the PlantTFDB v3.0 database (http://plntfdb.bio.uni-potsdam .de/v3.0/).
BUSCO v3.0.2 (Benchmarking Universal Single-Copy Orthologs) [25] was utilized to assess the completeness of the transcriptome assembly by using a core set of conservative orthologs in eukaryotic species from the OrthoDB database (https://busco.ezlab.org/datasets/).

2.3. Identification of Orthologous Genes, Phylogenetic Analysis, and Analysis of Genes under Positive Selection. OrthoFinder v2.3.3 was used to identify orthologs in four fern species (A. nidus, A. komarovii, A. formosae, and Goniophlebium niponicum), and transcriptome data were downloaded from the GigaScience repository, GigaDB $[26,27]$. To identify genes under positive selection in $A$. nidus, orthologroups with single-copy genes were retained for further phylogenetic analysis. Amino acid sequences of each orthologroups were aligned using MUSCLE v3.8.31 with default parameters [28]. Maximum likelihood trees were constructed using RAxML v8 with the PROTGAMMAIJTTF model based on the identified orthologous genes [29]. Further, we applied the improved branch-site model in codeml of the PAML v4.8 package on each orthogroups [30]. A. nidus was set as the foreground branch, and we calculated the rates of nonsynonymous substitutions (Ka) and synonymous substitutions (Ks). The likelihood ration test and chi-square test were applied to test for significance. $\mathrm{Ka}<\mathrm{Ks}$ suggested negative (purifying) selection, and $\mathrm{Ka}>\mathrm{Ks}$ indicated positive selection [31]. In this study, we retained genes with $\mathrm{Ks}>0.005$; genes with a $\mathrm{Ka} / \mathrm{Ks}$ ratio greater than 1 were considered putative positively selected genes.

2.4. Functional Enrichment. We performed GO enrichment analysis to examine the functional genes involved in the adaptive evolution of $A$. nidus. This analysis was performed in agriGO v2.0 using the singular enrichment analysis tool with hypergeometric's test, as well as by clusterProfiler in $\mathrm{R}$ software; Fisher's exact tests $(P<0.05)$ were used to identify genes that were enriched in $A$. nidus compared with $A$. komarovii $[32,33]$.

2.5. Identification and Phylogenetic Analysis of MADS-Box Family Genes. We identified putative MADS-box proteins in $A$. nidus and A. komarovii by hidden Markov model (HMM) analysis. Firstly, BLASTP was performed against the protein database of $A$. nidus or A. komarovii using Arabidopsis MADS-box proteins as queries; the cut-off $e$ value for these searches was $1 e-05$. Secondly, proteins with SRF-TF domains (PF00319) were obtained from the Pfam database, and a HMM analysis was used to identify members of the MADS-box TF family present in A. nidus or A. komarovii. We verified the identity of the MADS-box candidate genes using SMART (the Simple Modular Architecture Research Tool: http://smart.embl-heidelberg.de/) and the NCBI Conserved Domain Database. Sequences with incomplete MADSbox domains and redundant sequences (with identities higher than 99\%) were removed.

To clarify the evolutionary relationships among the MADS-box family genes, 107 Arabidopsis MADS-box coding sequences and 49 new candidate MADS-box genes identified in $A$. nidus and A. komarovii were aligned using ClustalX 
TABle 1: Summary statistics of the completeness assessments of A. nidus and A. komarovii transcriptome assemblies.

\begin{tabular}{lcccccc}
\hline ID & Species & $\begin{array}{c}\text { Total number of } \\
\text { clean reads (Mb) }\end{array}$ & $\begin{array}{c}\text { Total number } \\
\text { of unigenes }\end{array}$ & $\begin{array}{c}\text { Mean } \\
\text { length (bp) }\end{array}$ & $\begin{array}{c}\text { N50 } \\
\text { value (bp) }\end{array}$ & $\begin{array}{c}\text { BUSCOs notation } \\
\text { assessment results }\end{array}$ \\
\hline RS259A & Asplenium nidus & 44.04 & 89,741 & 770 & 1,314 & $\begin{array}{c}C: 68.3 \%[S: 32.4 \% D: 35.9 \%], \\
F: 13.5 \%, M: 18.2 \%, n: 429\end{array}$ \\
RS261A & Asplenium komarovii & 44.57 & 77,912 & 1,049 & 1,872 & $\begin{array}{c}\text { C: } 77.7 \%[S: 42.7 \%, D: 35.0 \%], \\
F: 7.5 \%, M: 14.8 \%, n: 429\end{array}$ \\
\hline
\end{tabular}

C: complete BUSCOs; S: complete and single-copy BUSCOs; D: complete and duplicated BUSCOs; F: fragmented BUSCOs; M: missing BUSCOs; n: total number of BUSCO groups searched.

v2.1 and a phylogenetic tree was constructed with MrBayes v3.2 using the mixed model (the number of generations as set to 10,000 and the sampling frequency was set to ten). We added generations and maintained the sampling frequency until the standard deviation of split frequencies was below 0.01 . The final model contained ten million generations and a tree sampling density of 10,000 generations [34]. The top $25 \%$ of samples was discarded as the burn-in. Thereafter, we constructed a phylogenetic tree using all candidate MADS-box protein sequences from $A$. nidus, $A$. komarovii, and Arabidopsis thaliana (Arabidopsis) containing a conserved MADS domain. Next, phylogeny of type II MADS-box proteins-which contained conserved MADS-, I, and K-domains-was analyzed.

\section{Results}

3.1. De Novo Assembly, Completeness Assessment, and Annotation. We sequenced the transcriptomes of $A$. nidus and $A$. komarovii by RNA-seq, and obtained 44.04 and $44.57 \mathrm{Mb}$ clean reads, respectively. Trinity was used for the de novo assembly of 173,229 and 142,138 contigs in $A$. nidus and A. komarovii transcriptomes (Table 1). After the redundancies were removed, these transcriptome assemblies yielded 89,741 and 77,912 unigenes in A. nidus and A. komarovii, with N50 values of $1,314 \mathrm{bp}$ and $1,872 \mathrm{bp}$, respectively.

We used BUSCO to assess the completeness and quality of the transcriptome assemblies by using a eukaryotic species database containing 429 orthologroups as the reference. These transcriptomes showed high coverage rates of the orthologroups-68.3\% in A. nidus and $77.7 \%$ in A. komarovii (Table 1)-indicating that the transcriptomes were relatively complete, and that the data was of high quality and could be used for subsequent analyses.

Functional annotation of the transcriptomes was performed using data from seven public databases. A total of $52,305(58.28 \%$ of the genes in the transcriptome) and 45,938 (58.96\%) unigenes were successfully annotated in $A$. nidus and A. komarovii, respectively (Table 2). Detailed information on the functional annotation is listed in Table 2.

3.2. Comparative Analysis of Unigenes. We compared the orthologous genes present in both $A$. nidus and A. komarovii. A total of 20,064 orthologroups were shared by these species, including 25,022 and 27,748 genes in each transcriptome. In addition, we identified 18,160 and 8,970 A. nidus- and A. komarovii-specific genes. Subsequently, we performed GO enrichment analysis of genes from $A$. nidus
TABLE 2: Functional annotation results of $A$. nidus and A. komarovii transcriptomes.

\begin{tabular}{lcccc}
\hline Database & \multicolumn{2}{c}{ Asplenium nidus } & \multicolumn{2}{c}{ Asplenium komarovii } \\
\hline Total & 89,741 & $100 \%$ & 77,912 & $100 \%$ \\
Overall & 52,305 & $58.28 \%$ & 45,938 & $58.96 \%$ \\
$\mathrm{Nt}$ & 25,472 & $28.38 \%$ & 25,050 & $32.15 \%$ \\
$\mathrm{Nr}$ & 47,800 & $53.26 \%$ & 41,178 & $52.85 \%$ \\
COG & 17,509 & $19.51 \%$ & 16,985 & $21.80 \%$ \\
Swiss-Prot & 33,121 & $36.91 \%$ & 28,148 & $36.13 \%$ \\
InterPro & 34,663 & $38.63 \%$ & 31,923 & $40.97 \%$ \\
GO & 17,871 & $19.91 \%$ & 14,801 & $19.00 \%$ \\
KEGG & 36,164 & $40.30 \%$ & 31,253 & $40.11 \%$ \\
\hline
\end{tabular}

Note: the two columns represent the number of unigenes and the percentage of annotated unigenes.

(Figure 1, Tables S1). We enriched 38 and 45 GO terms by clusterProfiler and agriGO in $A$. nidus $(P<0.05)$, respectively, in which 24 terms were enriched by both methods. For instance, the regulation of the response to stimulus GO term (GO: 0048583, 51 genes) was enriched only in A. nidus. This GO term covered six genes related to abscisic acid synthesis (ABA), and six genes associated with photosynthesis (Table S2). Taken together, our results revealed that the $A$. nidus-specific genes were mainly involved in the regulation of the response to stimulus and related to photosynthesis, stress tolerance, and ABA signalling.

Next, we identified TFs present in $A$. nidus and $A$. komarovii transcriptomes by querying sequences against PlantTFDB. A total of 1,528 and 1,198 putative TF coding unigenes were identified in $A$. nidus and A. komarovii, respectively (Figure 2 ).

Several TFs, such as LIM, TCP, zinc finger-related (including Zf-HD, C2C2-Dof, C2H2, and C3H), and MYBfamily TFs, were significantly more abundant in $A$. nidus than in A. komarovii. These TFs are mainly involved in leaf and root development, nitrogen assimilation, and plant stress response; therefore, they may be related to the adaptation of A. nidus to its epiphytic environments. Moreover, we found that the MADS-box family TFs were less abundant in $A$. nidus than in A. komarovii.

3.3. Genes under Positive Selection Were Annotated. We used the modified branch-site model in PAML to identify genes under positive selection from the 20,064 orthogroups. As a result, nine genes showed significant evidence of positive 


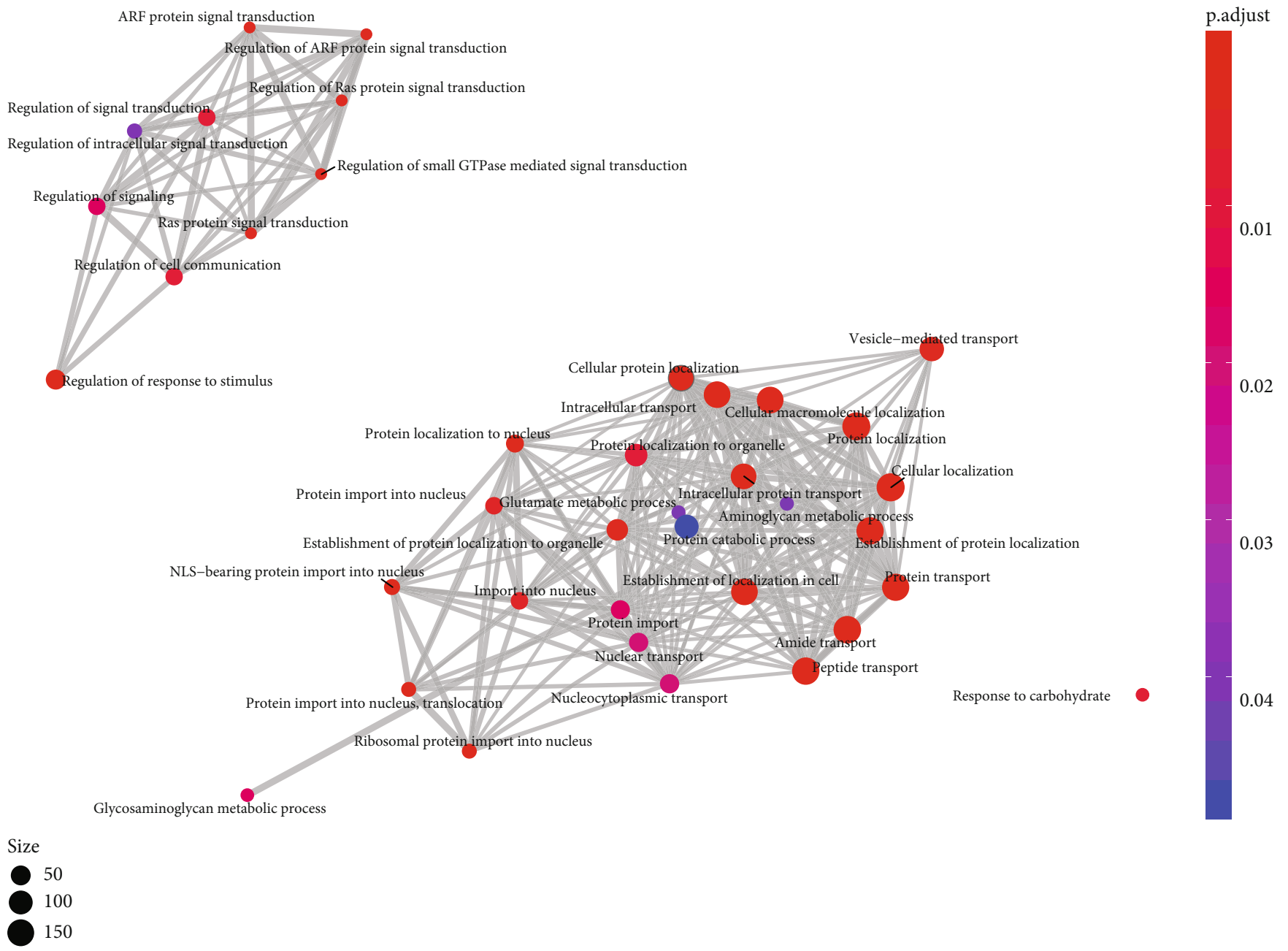

FIgURE 1: GO enrichment map for A. nidus. GO terms, visualized as dots, and shared genes are linked with gray lines.

selection (Table S3). These genes were mainly related to environmental adaptability, which included environmental responses and pressure stimulation (i.e., stress-associated protein CL11175 and zinc finger protein CL6726, genes involved in stress tolerance and photosynthesis (i.e., CL12383)).

\subsection{Identification and Phylogenetic Analysis of MADS-Box} Family Genes. With extremely specialized roots, $A$. nidus can readily colonize rainforest trees and maintain growth even in very dry conditions. In this way, the roots of $A$. nidus are very different from those of terrestrial ferns such as $A$. komarovii. In Arabidopsis, MADS-box genes in ANR1- and AGL12-subclades are involved in root development and differentiation [35]. Given the difference in the copy number of MADS-box family genes in A. nidus and A. komarovii, we identified and characterized MADS-box family genes in these two species. HMM analysis and manual searching identified 24 and 49 MADS-box family genes in A. nidus and A. komarovii, respectively. After removing the redundant sequences and genes with incomplete MADS-box domains, a total of 24 and 25 MADS-box family genes were subjected to further phylogenetic analyses. To clarify the evolutionary relationships among the MADS-box family genes, we constructed a phylogenetic tree using the amino acid sequences of MADS-box family genes in A. nidus, A. komarovii, and Arabidopsis. Phylogenetic analysis (Figure S1) revealed that one gene from $A$. komarovii clustered with $\mathrm{M} \alpha$-type Arabidopsis genes, but we did not find any type I MADSbox family genes in $A$. nidus. With respect to type II MADS-box genes, the MIKC proteins were further divided into 13 well-characterized subclades (Figure 3 ). One gene was identified as a MIKC*-type gene in A. komarovii. In addition, A. komarovii was found to contain four SVP subclade genes; however, these genes were absent in $A$. nidus. Interestingly, 24 genes in the ANR1 subclade were found to have expanded in A. nidus compared to 19 genes in A. komarovii.

\section{Discussion}

Epiphytes are unique plants that grow on other plants, and they are widely distributed in temperate and tropical rainforests $[1,5]$. A. nidus, the bird's nest fern is present in tropical 


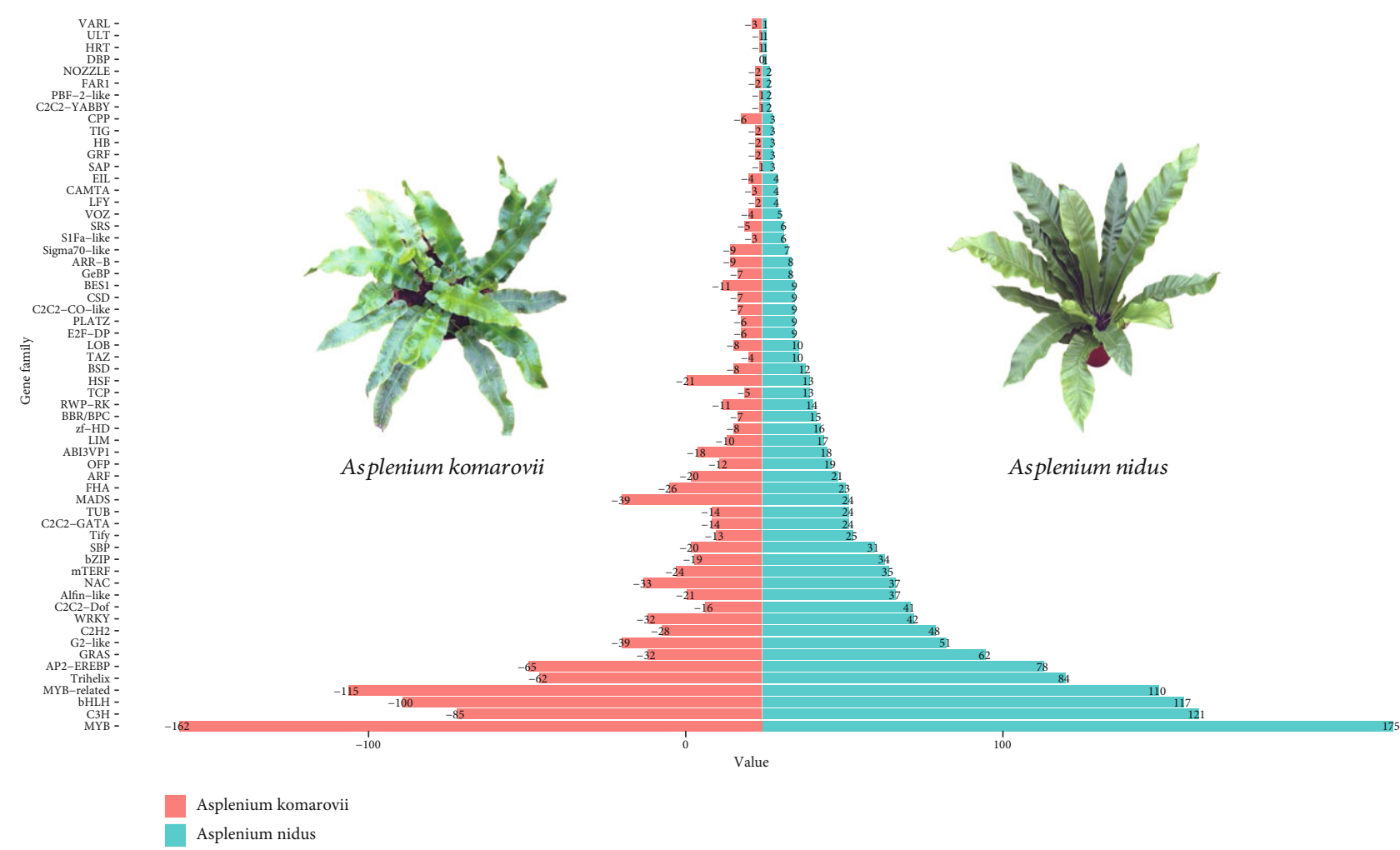

FIgure 2: The distribution of TF gene families in A. nidus and A. komarovii. Blue columns represent TF genes in A. nidus, and red columns represent TF genes in A. komarovii.

rainforests on tree trunks or rocks, whereas A. komarovii is a terrestrial species that thrives in temperature regions. $A$. nidus is considered to be more sensitive to climate fluctuations and could endure severe drought stress, nutrient shortage, and intense sunlight $[5,36,37]$. Several epiphytic ferns exhibit features typical of CAM photosynthesis-an adaptative mechanism to dry habitats [6-8]. Previous studies have suggested that large plants, thick fronds, and robust root systems are the major physiological adaptations to drought, although similar analyses of the adaptive features of pure C3 plants have not yet been performed [38-40]. In this study, we identified the factors affecting environmental adaptability in epiphytic $A$. nidus using transcriptomic data. First, we identified genes unique to $A$. nidus, including those related to the regulation of response to stimulus (GO: 0048583, 51 genes). These genes are mainly involved in abiotic stress tolerance, ABA, and photosynthesis; they may contribute to the adaptation of $A$. nidus to drought stress and intense sunlight. Second, we identified genes under positive selection. These genes are usually associated with adaptation [41]. We found nine genes under positive selection in $A$. nidus (Ka/Ks $>1$; Table S3). Among these genes, three were related to environmental adaptability, including stress-associated protein responses, as well as photosynthesis. Previous studies have shown that OsAKT1 plays essential roles in the ability of $\mathrm{K}+$ channels to uptake in rice; $\mathrm{K}$ has crucial roles in various physiological processes, including photosynthesis, assimilated products transport, and tolerance to biotic or abiotic stresses [35, 42]. These genes may also be responsible for the adaptation of $A$. nidus to extreme environments and intense sunlight. Comparisons of the TFs between these two species revealed a significant expansion of the zinc finger-related and MYB gene families in A. nidus (Figure 2). The zinc finger-related and MYB gene families were previously reported to be involved in drought tolerance via ABA signalling [43-45]. Given that these TF gene families involved in ABA signalling and plant responses to drought stress, it is likely that $A$. nidus utilizes ABA signalling-mediated pathways to adapt to the epiphytic lifestyle in the tropical rainforest. In addition, genes related to photosynthesis were identified in A. nidus by the functional annotation of specific genes and the analysis of genes under positive selection. These genes may contribute to the adaptation of $A$. nidus to intense sunlight [46].

Compared to terrestrial species that take root in soil, epiphytes colonize the crowns of forest trees and face challenges in obtaining water and nutrients [39]. A. nidus, a well-known epiphytic fern, has been hypothesized to endure dry conditions by making use of a unique root system and large fronds [38, 47]. It possesses sponge-like roots to absorb and store water, as well as a mass of scaly hairs to protect the apical meristem. In addition, it utilizes its fronds to form basket-shaped rosettes to intercept humus, generate suspended organic soil, and improve nutrient availability.

Significant expansion of gene families is known to be correlated with the adaptive evolution of closely related species $[48,49]$. To study the mechanisms that facilitate the absorption of water, we examined genes related to root and leaf development. Comparisons of TFs showed that members 


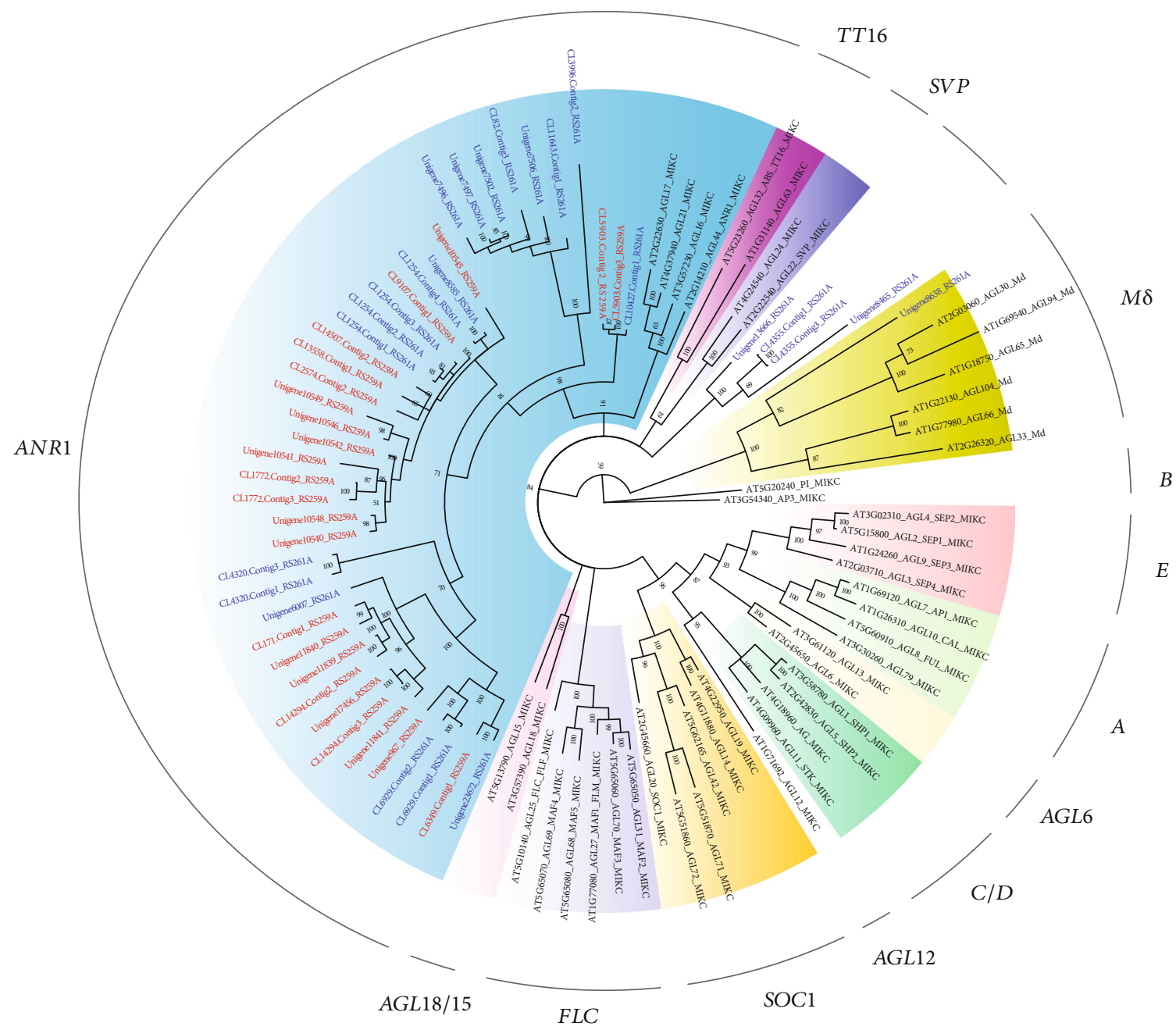

Figure 3: Phylogenetic tree of type II MADS-box proteins in Arabidopsis, A. nidus, and A. komarovii. At, Arabidopsis; RS259, A. nidus; RS261, A. komarovii. In total, 13 clades were formed and marked with different colours.

of the MADS-box, LIM, and TCP TF families varied considerably between $A$. nidus and A. komarovii. Specifically, the ANR1 subclade exhibited a considerable expansion in $A$. nidus (24 members) compared to A. komarovii (19 members). However, this conclusion should be cautious because of the transcriptome data used. The higher number of ANR1 genes in $A$. nidus may be related with its higher assembly completeness (52,305 and 45,938 in A. nidus and A. komarovii, respectively). Nevertheless, BUSCO results showed high coverage rates of the orthologroups in A. nidus. At this point, further analyses and complete data are needed to support our speculation. In seed plants, the ANR1 proteins regulate lateral root development $[50,51]$. The expanded ANR1-family genes identified here may have acquired novel root development functions via neofunctionalization. The LIM family has been reported to be associated with primary and lateral root development $[52,53]$. In addition, class II plant-specific TCP TFs are known to affect local patterns of cell proliferation and to control morphological traits that determine evolutionary success, including leaf formation and shoot branching [54]. These observations suggested that the expansion of gene families related to root or leaf development in A. nidus had facilitated its adaptation to drought. More importantly, it is apparent that the epiphytic A. nidus has evolved specific strategies with respect to photosynthesis, root development, and frond morphology to withstand extreme canopy environments.

\section{Conclusions}

Due to its epiphytic growth habitat, A. nidus has been forced to withstand extreme environments. In this study, we constructed de novo transcriptome assemblies for $A$. nidus and its close relative $A$. komarovii. Comparative transcriptomic analysis showed that genes unique to $A$. nidus were mainly involved in stress tolerance and photosynthesis, implying that these genes may contribute to its adaptation to drought stress and intense sunlight. It is notable that the expansion of TF gene families and A. nidus-specific genes was related to $\mathrm{ABA}$ signalling pathway and stress responses, which potentially reflect the adaptation of $A$. nidus to drought. The expansion of TF gene families related to root or leaf 
development may have also facilitated this adaptation. Overall, our data suggest that $A$. nidus has evolved specific adaptations related to photosynthesis, root development, and large frond morphology to withstand the extreme epiphytic environment. There are also important limitations to our study. Firstly, the transcriptome represented only a portion of all coding genes; therefore, it provided limited sequence information due to the fact that genes are dynamically expressed. Secondly, tissues of A. nidus and other species should be sampled to verify these findings. Lastly, more detailed functional experiments are needed to provide deeper insights into the molecular mechanisms of epiphytic adaptations to harsh environments.

\section{Data Availability}

The data used to support the findings of this study have been deposited in the NCBI short reads archive repository under accession numbers SAMN11175064 (A. nidus) and SAMN11175065 (A. komarovii).

\section{Conflicts of Interest}

The authors declare that there is no conflict of interest regarding the publication of this paper.

\section{Authors' Contributions}

Yue-Hong Yan, Rui Zhang, and Jiao Zhang conceived and designed this study. Hui Shen collected plant specimens. Bioinformatics analyses were performed by Jiao Zhang, Li Liu, and Jiang-Ping Shu. Jiao Zhang, Rui Zhang, Dong-Mei Jin, Hong-Feng Chen, and Yue-Hong Yan drafted the manuscript.

\section{Acknowledgments}

We thank Mr. Hui Shang from the Shanghai Chenshan Botanical Garden of China and Prof. Paul G. Wolf from Utah State University for providing valuable advices. This study was supported by the Shanghai Landscaping \& City Appearance Administrative Bureau of China (grant numbers G192421 and G182410), the Strategic Priority Research Program of the Chinese Academy of Sciences (XDA13020603), and the National Natural Science Foundation of China (No. 31800450).

\section{Supplementary Materials}

Supplementary 1. Figure S1: the phylogenetic tree of MADSbox genes in Arabidopsis thaliana, Asplenium nidus, and Asplenium komarovii constructed with MrBayes.

Supplementary 2. Table S1: the enrichment of specific genes in $A$. nidus.

Supplementary 3. Table S2: the functional annotation of specific genes in $A$. nidus.

Supplementary 4. Table S3: GO annotation of positively selected genes in $A$. nidus.

\section{References}

[1] D. H. Benzing, Vascular Epiphytes: General Biology and Associated Biota, Cambridge University Press, Cambridge, 1990.

[2] G. Zotz and P. Hietz, "The physiological ecology of vascular epiphytes: current knowledge, open questions," Journal of Experimental Botany, vol. 52, no. 364, pp. 2067-2078, 2001.

[3] S. F. Bartels and H. Y. H. Chen, "Mechanisms Regulating Epiphytic Plant Diversity," Critical Reviews in Plant Sciences, vol. 31, no. 5, pp. 391-400, 2012.

[4] N. M. Nadkarni, "Canopy roots: convergent evolution in rainforest nutrient cycles," Science, vol. 214, no. 4524, pp. 1023-1024, 1981.

[5] J. Dubuisson, H. Schneider, and S. Hennequin, "Epiphytisme chez les fougeres : diversite et histoire," Comptes Rendus Biologies, vol. 332, no. 2-3, pp. 120-128, 2009.

[6] J. A. M. Holtum and K. Winter, "Degrees of crassulacean acid metabolism in tropical epiphytic and lithophytic ferns," Australian Journal of Plant Physiology, vol. 26, no. 8, pp. 749-757, 1999.

[7] B. D. Minardi, A. P. L. Voytena, M. Santos, and Á. M. Randi, "Water stress and abscisic acid treatments induce the CAM pathway in the epiphytic fern Vittaria lineata (L.) Smith," Photosynthetica, vol. 52, no. 3, pp. 404-412, 2014.

[8] G. Rut, J. Krupa, Z. Miszalski, A. Rzepka, and I. Ślesak, "Crassulacean acid metabolism in the epiphytic fern Patycerium bifurcatum," Photosynthetica, vol. 46, no. 1, pp. 156-160, 2008.

[9] D. J. Ohlsen, L. R. Perrie, L. D. Shepherd, P. J. Brownsey, and M. J. Bayly, "Phylogeny of the fern family Aspleniaceae in Australasia and the south-western Pacific," Australian Systematic Botany, vol. 27, no. 6, pp. 355-371, 2015.

[10] E. Schuettpelz and K. M. Pryer, "Evidence for a Cenozoic radiation of ferns in an angiosperm-dominated canopy," Proceedings of the National Academy of Sciences of the United States of America, vol. 106, no. 27, pp. 11200-11205, 2009.

[11] K.-. W. Xu, L. Zhang, C. J. Rothfels et al., "A global plastid phylogeny of the fern genus Asplenium (Aspleniaceae)," Cladistics, 2019.

[12] X. Li, H. X. Wu, and S. G. Southerton, "Comparative genomics reveals conservative evolution of the xylem transcriptome in vascular plants," BMC Evolutionary Biology, vol. 10, no. 1, p. 190, 2010.

[13] A. Necsulea and H. Kaessmann, "Evolutionary dynamics of coding and non-coding transcriptomes," Nature Reviews. Genetics, vol. 15, no. 11, pp. 734-748, 2014.

[14] I. G. Romero, I. Ruvinsky, and Y. Gilad, "Comparative studies of gene expression and the evolution of gene regulation," Nature Reviews. Genetics, vol. 13, no. 7, pp. 505-516, 2012.

[15] C. Palma-Silva, M. Ferro, M. Bacci, and A. C. Turchetto-Zolet, "De novo assembly and characterization of leaf and floral transcriptomes of the hybridizing bromeliad species (Pitcairnia spp.) adapted to Neotropical Inselbergs," Molecular Ecology Resources, vol. 16, no. 4, pp. 1012-1022, 2016.

[16] S. Sudheesh, T. I. Sawbridge, N. O. I. Cogan, P. Kennedy, J. W. Forster, and S. Kaur, "De novo assembly and characterisation of the field pea transcriptome using RNA-Seq," BMC Genomics, vol. 16, no. 1, p. 611, 2015.

[17] Z. Xia, H. Xu, J. Zhai et al., "RNA-Seq analysis and de novo transcriptome assembly of Hevea brasiliensis," Plant Molecular Biology, vol. 77, no. 3, pp. 299-308, 2011. 
[18] P. Chomczynski and N. Sacchi, "The single-step method of RNA isolation by acid guanidinium thiocyanate- phenolchloroform extraction: twenty-something years on," Nature Protocols, vol. 1, no. 2, pp. 581-585, 2006.

[19] M. P. Cox, D. A. Peterson, and P. J. Biggs, "SolexaQA: ata-glance quality assessment of Illumina second-generation sequencing data," BMC Bioinformatics, vol. 11, no. 1, p. $485,2010$.

[20] B. J. Haas, A. Papanicolaou, M. Yassour et al., "De novo transcript sequence reconstruction from RNA-seq using the Trinity platform for reference generation and analysis," Nature Protocols, vol. 8, no. 8, pp. 1494-1512, 2013.

[21] G. Pertea, X. Huang, F. Liang et al., "TIGR gene indices clustering tools (TGICL): a software system for fast clustering of large EST datasets," Bioinformatics, vol. 19, no. 5, pp. 651-652, 2003.

[22] E. Quevillon, V. Silventoinen, S. Pillai et al., "InterProScan: protein domains identifier," Nucleic Acids Research, vol. 33, pp. W116-W120, 2005.

[23] A. Conesa, S. Gotz, J. M. Garcia-Gomez, J. Terol, M. Talon, and M. Robles, "Blast2GO: a universal tool for annotation, visualization and analysis in functional genomics research," Bioinformatics, vol. 21, no. 18, pp. 3674-3676, 2005.

[24] R. Finn, J. Clements, and S. R. Eddy, "HMMER web server: interactive sequence similarity searching," Nucleic Acids Research, vol. 39, no. 2, pp. W29-W37, 2011.

[25] F. A. Simão, R. M. Waterhouse, P. Ioannidis, E. V. Kriventseva, and E. M. Zdobnov, "BUSCO: assessing genome assembly and annotation completeness with single-copy orthologs," Bioinformatics, vol. 31, no. 19, pp. 3210-3212, 2015.

[26] D. Emms and S. L. Kelly, "OrthoFinder: solving fundamental biases in whole genome comparisons dramatically improves orthogroup inference accuracy," Genome Biology, vol. 16, no. 1, p. 157, 2015.

[27] H. Shen, D. Jin, J.-P. Shu et al., "Large-scale phylogenomic analysis resolves a backbone phylogeny in ferns," GigaScience, vol. 7, no. 2, pp. 1-11, 2018.

[28] R. C. Edgar, "MUSCLE: multiple sequence alignment with high accuracy and high throughput," Nucleic Acids Research, vol. 32, no. 5, pp. 1792-1797, 2004.

[29] A. Stamatakis, "RAxML version 8: a tool for phylogenetic analysis and post-analysis of large phylogenies," Bioinformatics, vol. 30, no. 9, pp. 1312-1313, 2014.

[30] Z. Yang, "PAML: a program package for phylogenetic analysis by maximum likelihood," Bioinformatics, vol. 13, no. 5, pp. 555-556, 1997.

[31] L. D. Hurst, "The Ka/Ks ratio: diagnosing the form of sequence evolution," Trends in Genetics, vol. 18, no. 9, pp. 486-487, 2002.

[32] T. Tian, Y. Liu, H. Yan et al., "agriGO v2.0: a GO analysis toolkit for the agricultural community, 2017 update," Nucleic Acids Research, vol. 45, no. W1, pp. W122-W129, 2017.

[33] G. Yu, L. Wang, Y. Han, and Q. Y. He, “clusterProfiler: an R package for comparing biological themes among gene clusters," OMICS, vol. 16, no. 5, pp. 284-287, 2012.

[34] F. Ronquist and J. P. Huelsenbeck, "MrBayes 3: Bayesian phylogenetic inference under mixed models," Bioinformatics, vol. 19, no. 12, pp. 1572-1574, 2003.

[35] Y. Wang and W. Wu, "Potassium transport and signaling in higher plants," Annual Review of Plant Biology, vol. 64, no. 1, pp. 451-476, 2013.
[36] M. S. R. Adibah and A. N. Ainuddin, "Epiphytic plants responses to light and water stress," Asian Journal of Plant Sciences, vol. 10, no. 2, pp. 97-107, 2011.

[37] N. A. Ainuddin and D. A. N. Najwa, "Growth and physiological responses of Asplenium nidus to water stress," Asian Journal of Plant Sciences, vol. 8, no. 6, pp. 447-450, 2009.

[38] M. Freiberg and S. M. Turton, "Importance of drought on the distribution of the birds nest fern, Asplenium nidus, in the canopy of a lowland tropical rainforest in north-eastern Australia," Austral Ecology, vol. 32, no. 1, pp. 70-76, 2007.

[39] M. Kluge, P. N. Avadhani, and C. J. Goh, "Gas Exchange and Water Relations in Epiphytic Tropical Ferns," Ecological Studies, vol. 76, pp. 87-108, 1989.

[40] G. Zotz, P. Hietz, and G. Schmidt, "Small plants, large plants: the importance of plant size for the physiological ecology of vascular epiphytes," Journal of Experimental Botany, vol. 52, no. 363, pp. 2051-2056, 2001.

[41] Z. Zhang, Z. He, S. Xu et al., "Transcriptome analyses provide insights into the phylogeny and adaptive evolution of the mangrove fern genus Acrostichum," Scientific Reports, vol. 6, article 35634, 2016.

[42] L. Wu, F. Holtkamap, A. Wairirich, and M. Frei, "Potassium Ion Channel Gene OsAKT1 Affects Iron Translocation in Rice Plants Exposed to Iron Toxicity," Frontiers in Plant Science, vol. 10, p. 579, 2019.

[43] M. Farooq, A. Wahid, N. Kobayashi, D. Fujita, and S. M. A. Basra, "Plant drought stress: effects, mechanisms and management," Agronomy for Sustainable Development, vol. 29, no. 1, pp. 185-212, 2009.

[44] A. Katiyar, S. Smita, S. K. Lenka, R. Rajwanshi, V. Chinnusamy, and K. Bansal, "Genome-wide classification and expression analysis of $M Y B$ transcription factor families in rice and Arabidopsis," BMC Genomics, vol. 13, no. 1, p. 544, 2012.

[45] H. Takatsuji, "Zinc-finger transcription factors in plants," Cellular and Molecular Life Sciences, vol. 54, no. 6, pp. 582-596, 1998.

[46] R. P. Sinha and D. Hader, "UV-induced DNA damage and repair: a review," Photochemical and Photobiological Sciences, vol. 1, no. 4, pp. 225-236, 2002.

[47] T. M. Fayle, A. Y. C. Chung, A. J. Dumbrell, P. Eggleton, and W. A. Foster, "The effect of rain forest canopy architecture on the distribution of epiphytic ferns (Asplenium spp.) in Sabah, Malaysia," Biotropica, vol. 41, no. 6, pp. 676-681, 2009.

[48] P. H. Sudmant, J. O. Kitzman, F. Antonacci et al., "Diversity of human copy number variation and multicopy genes," Science, vol. 330, no. 6004, pp. 641-646, 2010.

[49] T. Zhang, Q. Qiao, P. Y. Novikova et al., "Genome of Crucihimalaya himalaica, a close relative of Arabidopsis, shows ecological adaptation to high altitude," Proceedings of the National Academy of Sciences of the United States of America, vol. 116, no. 14, pp. 7137-7146, 2019.

[50] G. Zhang, K. Liu, Z. Li et al., "The Apostasia genome and the evolution of orchids," Nature, vol. 549, no. 7672, pp. 379383, 2017.

[51] H. Zhang and B. G. Forde, "An Arabidopsis MADS box gene that controls nutrient-induced changes in root architecture," Science, vol. 279, no. 5349, pp. 407-409, 1998.

[52] D. Arnaud, A. Déjardin, J. C. Leplé, M. C. Lesage-Descauses, and G. Pilate, "Genome-wide analysis of LIM gene family in 
Populus trichocarpa, Arabidopsis thaliana, and Oryza sativa," DNA Research, vol. 14, no. 3, pp. 103-116, 2007.

[53] M. Zhao, L. He, Y. Gu, Y. Wang, Q. Chen, and C. He, "Genome-wide analyses of a plant-specific LIM-domain gene family implicate its evolutionary role in plant diversification," Genome Biology and Evolution, vol. 6, no. 4, pp. 10001012, 2014.

[54] M. Martín-Trillo and P. Cubas, “TCP genes: a family snapshot ten years later," Trends in Plant Science, vol. 15, no. 1, pp. 3139,2010 . 


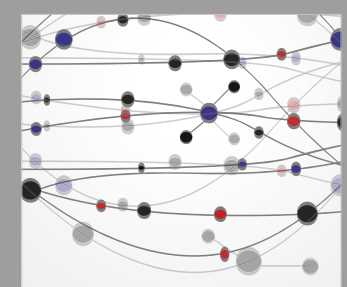

The Scientific World Journal
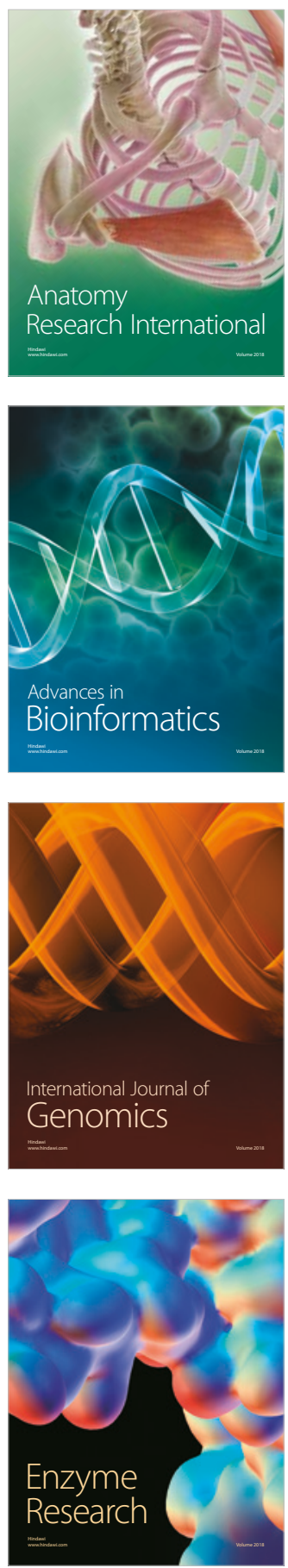
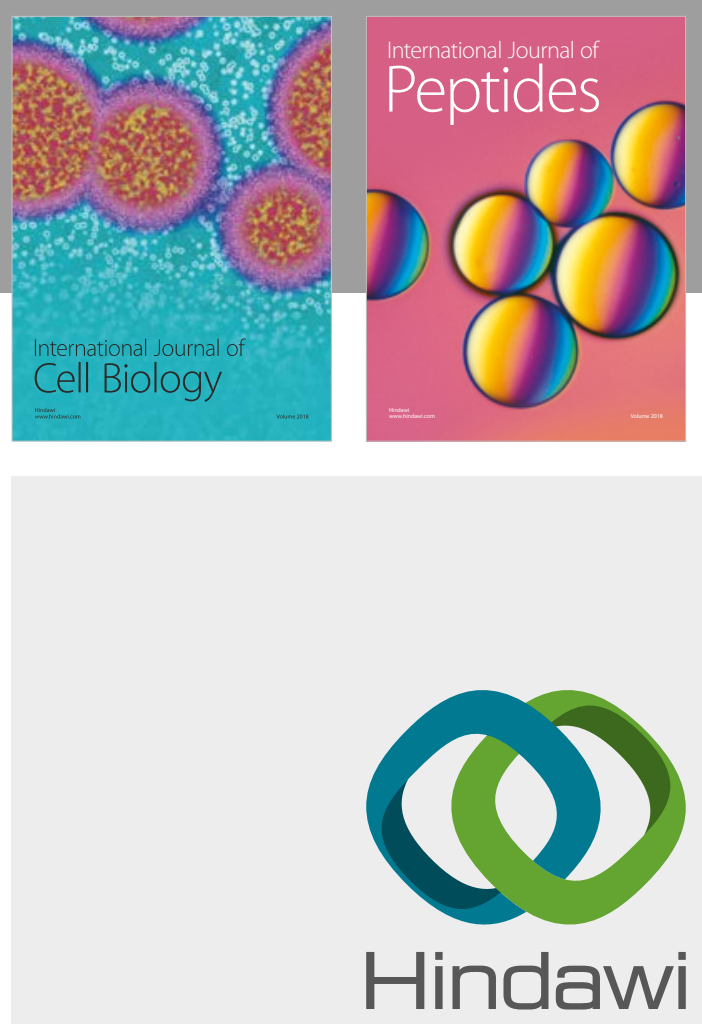

Submit your manuscripts at

www.hindawi.com
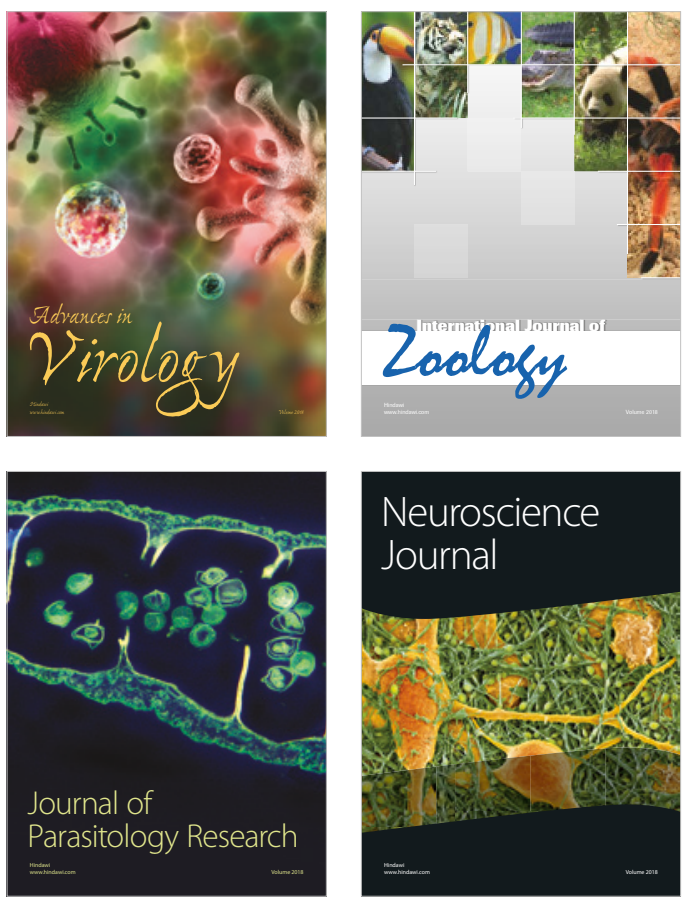
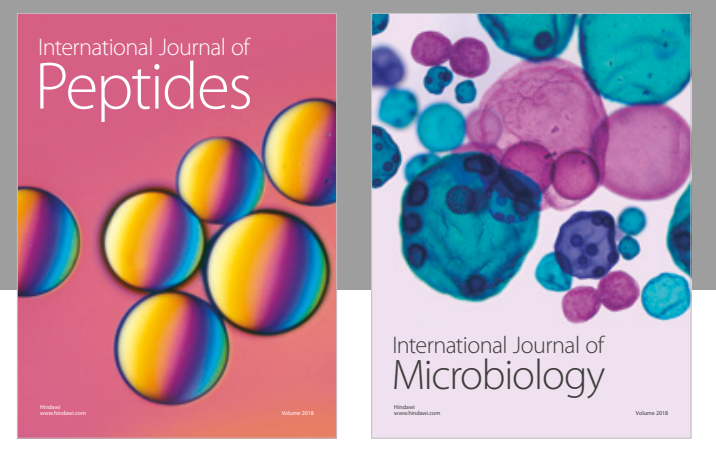

nternational Journal of Microbiology
Journal of
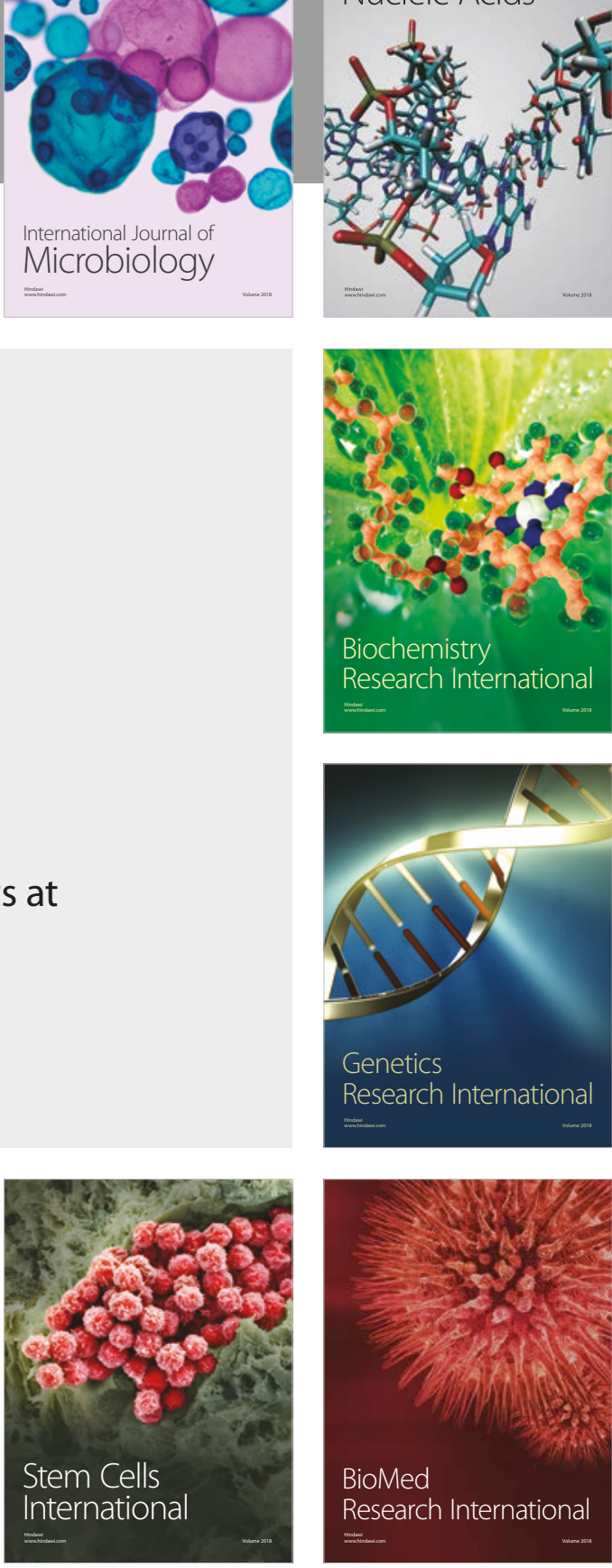
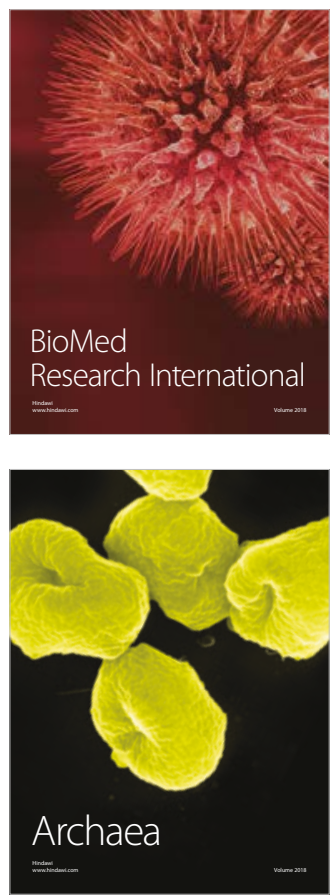